\title{
Modelagem da satisfação e identificação de atributos de qualidade em serviços odontológicos
}

\author{
Rafael Targanski ${ }^{\text {** }}$, Maria Auxiliadora Cannarozzo Tinoco ${ }^{\mathrm{b}}$, José Luis Duarte Ribeiroc \\ a*targanski@gmail.com, UFRGS, Brasil \\ bmaria@producao.ufrgs.br, UFRGS, Brasil \\ cribeiro@producao.ufrgs.br, UFRGS, Brasil
}

\begin{abstract}
Resumo
Este artigo apresenta a construção de um modelo de satisfação e a identificação e hierarquização dos atributos que afetam a qualidade percebida dos clientes de serviços odontológicos. Para atingir os objetivos, foram aplicadas duas pesquisas que permitiram identificar (i) as relações significativas entre os determinantes de satisfação e (ii) a hierarquia dos atributos de qualidade. A principal contribuição do artigo é a proposição de um modelo que pode auxiliar gestores de serviços odontológicos na melhoria da qualidade nos serviços e da satisfação de seus clientes, contribuindo para o crescimento das organizações e para as pesquisas do setor de serviços.
\end{abstract}

Palavras-chave

Satisfação de clientes. Qualidade percebida. Serviços odontológicos.

\section{Introdução}

Nos últimos anos a economia global atravessa um período de fortes mudanças no que tange à produção de bens, o gerenciamento de atividades e os serviços demandados. Essas novas mudanças trazem à tona a necessidade de uma gestão por desempenho e novas medidas ou indicadores para melhor controle de gestão (MüllER, 2003).

Juntamente com essas mudanças, o setor de serviços vem ocupando posição de destaque na economia mundial. Em 2001, nos Estados Unidos, os serviços empregavam cerca de $80 \%$ dos trabalhadores e, durante os últimos anos, observa-se uma importante evolução na economia global, que deixou de ser baseada em manufatura para ser predominantemente baseada em serviços e atendimento a clientes (RUST; ZEITHAML; LEMON, 2001). A mesma tendência é observada no Brasil, onde o setor de serviços ocupa posição de destaque na economia do país. Em 2005, a representação desse setor na economia brasileira em termos de empregos foi de $59,10 \%$ e a sua participação no PIB do país correspondeu a 65\% do total (INSTITUTO..., 2005).
A introdução de novas tecnologias, o crescimento da população e o aumento da qualidade de vida estão entre os fatores que contribuem significativamente para o crescimento do setor de serviços (GIANESI; CORREA, 1994). Dessa forma, novos tipos de clientes têm surgido, assim como tem crescido a importância da qualidade e da satisfação desses clientes para a competitividade dos fornecedores de serviços.

$\mathrm{Na}$ área da saúde, com o aumento da competitividade, as empresas têm sido obrigadas a alterar o seu comportamento frente aos clientes. Como consequência, elas têm buscado constantemente, através da análise de seu negócio, melhorar a qualidade dos serviços prestados. Assim, os métodos de avaliação de desempenho junto aos clientes têm sido aperfeiçoados, a fim de torná-los mais eficazes e eficientes (EINLOFT, 2004).

A qualidade percebida do serviço e a satisfação do cliente são conceitos diferentes, mas relacionados entre sim (OLIVER, 2010). A qualidade percebida do serviço é definida como a avaliação do cliente em relação à excelência global do serviço, enquanto a satisfação do cliente é vista como uma resposta 
emocional de realização associada a uma experiência de consumo (OLIVER, 2010). Pesquisas demonstram que a qualidade percebida é um determinante importante da satisfação do cliente, e juntas, por sua vez, afetam as atitudes dos clientes e os lucros das empresas (e.g., MARTIN et al., 2008; VLACHOS; VRECHOPOULOS, 2008; OLIVER, 2010).

Dessa maneira, considerando-se a satisfação e a qualidade como definidores do sucesso das organizações e também a importância e crescimento do setor de serviços, impõe-se a necessidade de se definirem os principais determinantes que afetam a satisfação de clientes e a percepção da qualidade na prestação de serviços (TINOCO, 2006). 0 estudo sobre os determinantes de satisfação e atributos de qualidade de serviços justifica-se não somente pela importância do assunto na atualidade empresarial, mas também por fornecer uma ferramenta para que empresas de serviços odontológicos consigam melhorar a percepção de qualidade e, em consequência, a satisfação dos clientes, direcionando os esforços para aqueles atributos e determinantes que mais importam para o cliente.

Neste contexto, o presente artigo tem por intuito a modelagem das relações entre os determinantes da satisfação dos clientes e a determinação da importância dos atributos mais importantes que afetam a percepção de qualidade em serviços odontológicos. Para isso, o artigo está estruturado da seguinte forma: na primeira seção é apresentada uma introdução ao assunto, seguida de um referencial teórico que aborda os tópicos satisfação de clientes, qualidade e serviços odontológicos. Na terceira seção são apresentados os procedimentos metodológicos. A quarta seção apresenta e discute os resultados obtidos. Por fim, são apresentadas as considerações finais do trabalho.

\section{Satisfação do cliente}

A satisfação do cliente é geralmente definida como uma função das percepções do cliente e das suas expectativas prévias. Deste encontro surgem os primeiros desafios, porque as expectativas e as percepções são dinâmicas por serem essencialmente subjetivas e mutantes e, portanto, difíceis de avaliar (RUSSO, 2003). A satisfação é definida também como a avaliação feita pelo cliente em relação ao atendimento ou não das suas necessidades e expectativas. A insatisfação do cliente seria gerada pela falha da empresa em ir ao encontro das mesmas necessidades e expectativas dos clientes (ZEITHAML, 2003).

Para justificar a busca pela satisfação do cliente, Bateson e Hoffman (2001) citam três razões fundamentais: os custos são maiores para obter novos clientes do que manter clientes antigos, a demanda competitiva das empresas por satisfação e o valor do ciclo de vida dos clientes.

Diversos modelos (OLIVER, 1980, 1993; SPRENG; MACKENZIE; OLSHAVSKY, 1996) buscam explicar a formação da satisfação dos clientes. Esses modelos foram criados nos últimos anos com a finalidade de relacionar os determinantes que afetam essa satisfação. Dentre eles, o modelo de desconfirmação das expectativas, proposto por Oliver (1980), é um dos mais citados na literatura para explicar a satisfação dos clientes (TINOCO, 2006).

0 modelo proposto por Oliver (1980, 2010), expresso na Figura 1, traz o paradigma da desconfirmação de expectativas como ideia central, onde a satisfação é resultante de um processo comparativo entre as expectativas iniciais dos clientes com os resultados decorrentes da compra do produto ou serviço. Pesquisas subsequentes buscaram estabelecer outras relações considerando um número maior de variáveis, tais como a influência dos afetos (OLIVER, 1993; MARTIN et al., 2008), dos desejos (SPRENG; MACKENZIE; OLSHAVSKY, 1996) e de outras variáveis como qualidade percebida (VLACHOS; VRECHOPOULOS, 2008; LIANG; ZHANG, 2009; HU; KANDAMPULLY; JUWAHEER, 2009), valor (HU; KANDAMPULLY; JUWAHEER, 2009), preço (LIANG; ZHANG, 2009) e imagem (LAl; GRIFFIN; BABIN, 2009) na formação da satisfação dos clientes.

Tinoco e Ribeiro (2007) propõem uma metodologia para a construção de modelos causais de satisfação de clientes de serviços seguindo procedimentos relativamente simples, que não requerem grandes amostras e permitem avaliar as relações existentes entre múltiplas variáveis. Os determinantes utilizados na modelagem proposta por esses autores são: imagem corporativa, desconfirmação de expectativas, emoções, desejos, expectativas, qualidade percebida, valor percebido, satisfação e preço do serviço. Tinoco, Araújo e Ribeiro (2007) aplicaram a abordagem proposta por Tinoco e Ribeiro (2007) ao setor de serviços médicos com o objetivo de identificar a relação entre os determinantes da satisfação neste setor, gerando o modelo de causa e efeito apresentado na Figura 2.

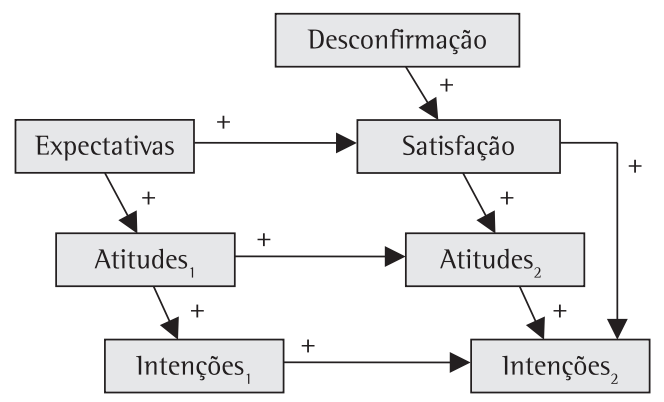

Figura 1. Modelo de desconfirmação de expectativas. Fonte: adaptado de Oliver (1980). 
O Quadro 1 apresenta as definições dos determinantes que são incluídos no modelo de satisfação de clientes do serviço médico, seguindo a abordagem para a modelagem da satisfação proposta por Tinoco e Ribeiro (2007).

\section{Qualidade em serviços}

A qualidade em serviços pode ser definida como a avaliação que o cliente faz, durante ou após o término do processo, através da comparação entre o que o cliente esperava do serviço e o que ele percebeu do serviço prestado (GIANESI; CORRÊA, 1994). Segundo Zeithaml (2003), a definição de qualidade segue uma visão de superioridade ou excelência. Para Grönroos (2003), qualidade em serviços deve refletir, acima de tudo, aquilo que os clientes percebem.

Parasuraman, Zeithaml e Berry (1985) foram dos primeiros autores a identificar dimensões de qualidade de serviços. Eles propõem um modelo para a medição dessa qualidade baseado na comparação entre o serviço percebido e o serviço esperado. Um dos resultados da pesquisa realizada por esses autores é que os clientes utilizam praticamente os mesmos critérios para julgar a qualidade de serviços, independente do tipo de serviço prestado. Assim, esses critérios foram agrupados em dez categorias, definidas como atributos ou determinantes da qualidade: acessibilidade, comunicação, competência, cortesia, credibilidade, confiabilidade, presteza, segurança, tangiveis e compreensão e conhecimento do cliente.

De maneira geral, os principais atributos de qualidade citados por diversos autores (PARASURAMAN; ZEITHAML; BERRY, 1985; GIANESI; CORRÊA, 1994; GRÖNROOS, 2003; MIGUEL; SALOMI, 2004; LADHARI, 2009) circundam os mesmos aspectos e podem ser consolidados, reduzindo-os a cinco atributos de qualidade mais abrangentes: confiabilidade (habilidade de prestar o serviço de forma confiável, precisa e consistente), presteza (disposição de prestar 0 serviço prontamente e auxiliar os clientes), confiança (conhecimento e cortesia dos funcionários e sua

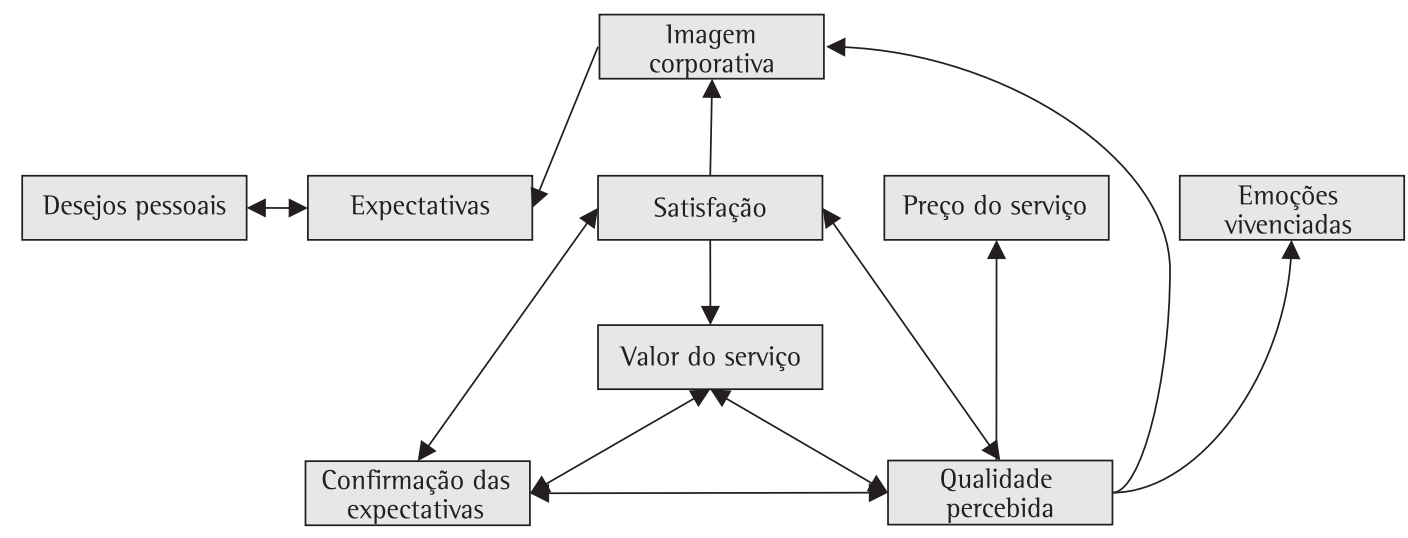

Figura 2. Modelo de causa e efeito da satisfação dos clientes de serviços médicos. Fonte: adaptado de Tinoco, Araújo e Ribeiro (2007).

Quadro 1. Definições dos determinantes de satisfação avaliados.

\begin{tabular}{|c|c|}
\hline Determinantes & Definição \\
\hline Desejos pessoais & $\begin{array}{l}\text { Afã de obter mais satisfação do que é efetivamente necessário. Condição psicológica/social insatisfatória que } \\
\text { nos leva a uma ação para remediá-la. }\end{array}$ \\
\hline Expectativas & $\begin{array}{l}\text { Crença prévia sobre o que o serviço irá oferecer. Construída a partir de experiências anteriores com o serviço, } \\
\text { informação recebida por propaganda e comunicação boca a boca. }\end{array}$ \\
\hline $\begin{array}{l}\text { Emoções vivenciadas no } \\
\text { momento }\end{array}$ & $\begin{array}{l}\text { Sentimentos positivos (ou negativos) vivenciados no momento da prestação de serviço. Sentimentos de } \\
\text { felicidade, prazer, entusiasmo, alegria, bom humor (ou mau humor, tristeza, pesar, arrependimento, raiva, } \\
\text { culpa). }\end{array}$ \\
\hline Qualidade percebida & $\begin{array}{l}\text { Avaliação da excelência do serviço em relação às dimensões de confiabilidade, responsabilidade, segurança, } \\
\text { empatia e tangíveis, conforme a percepção do cliente. }\end{array}$ \\
\hline Preço do serviço & Aquilo que é pago pelo serviço. Aquilo ao qual se renuncia ou que é sacrificado na obtenção de um serviço. \\
\hline Valor do serviço & Relação entre os benefícios obtidos e o preço do serviço (custo-benefício). \\
\hline Confirmação das expectativas & Comparação entre o que esperávamos receber e o que percebemos que recebemos do serviço. \\
\hline Imagem corporativa & Percepção em relação à corporação ou empresa de serviços, fixada na memória dos consumidores. \\
\hline Satisfação dos clientes & Estado afetivo gerado pela reação emocional à experiência com um serviço. \\
\hline
\end{tabular}

Fonte: adaptado de Tinoco (2006). 
habilidade de transmitir confiança, segurança e credibilidade), empatia (fornecimento de atenção individualizada aos clientes, facilidade de contato e comunicação) e tangíveis (aparência das instalações, equipamentos, funcionários e dos materiais de comunicação).

A avaliação da qualidade em serviços é um desafio, devido ao fato de a satisfação dos clientes ser determinada por fatores intangíveis. Diferentemente de produtos que possuem características mensuráveis de modo objetivo, a qualidade em serviços possui características psicológicas, que muitas vezes se estendem além da prestação do serviço, como no caso de serviços de saúde (FITZSIMMONS; FITZSIMMONS, 2000).

A identificação dos critérios pelos quais os clientes avaliam serviços é uma maneira de compreender melhor as expectativas dos clientes. A compreensão dos critérios priorizados pelos clientes para um determinado serviço permite que a gestão das operações em serviço possa garantir o desempenho adequado nesses critérios (GIANESI; CORRÊA, 1994).

No trabalho de Parasuraman, Zeithaml e Berry (1985) é proposto um modelo de avaliação da qualidade em serviços, o qual foi denominado de SERVQUAL . Esse modelo define que a qualidade é avaliada pelo consumidor na comparação entre suas expectativas e a percepção com relação ao desempenho do serviço, baseando-se em cinco atributos para a avaliação da qualidade, conforme citado anteriormente: confiabilidade, presteza, confiança, empatia e tangíveis.

Entretanto, Cronin e Taylor (1992) criticam a existência de cinco atributos genéricos quando se utiliza a escala SERVQUAL para serviços em setores diferentes e, após um estudo com diferentes tipos de serviços, concluem que os atributos da qualidade utilizados pelo instrumento não se aplicam a todos os tipos de serviços. Conforme a pesquisa de Cronin e Taylor (1992), o modelo SERVQUAL não foi confirmado para duas das quatro indústrias de serviços pesquisadas (serviços de controle de peste e de lavagem a seco). Esses autores propuseram então o instrumento SERVPERF como alternativa ao SERVQUAL, baseado somente na percepção de performance dos serviços. Segundo os mesmos autores, a escala SERVQUAL é inadequada, pois não há evidências teóricas ou empíricas que suportem a importância da relação expectativa-percepção como medida de qualidade de serviço, sendo que, para serviços, medidas de qualidade baseadas apenas no desempenho do serviço seriam mais adequadas.

Embora o instrumento SERVQUAL seja válido para compreender a forma como os clientes avaliam o serviço, propõe atributos ou dimensões que são excessivamente abrangentes para ajudar os responsáveis pela gestão das operações de serviços a tomar decisões que tornem o serviço mais competitivo aos olhos dos consumidores (GIANESI; CORRÊA, 1994). Uma das principais críticas ao modelo SERVQUAL é que não existe um conjunto de fatores genéricos que sejam importantes em todos os segmentos de serviços (SCHNEIDER; WHITE, 2004). As generalizações são difíceis de fazer devido às diferenças na natureza de cada tipo de serviço (CHOWDHARY; PRAKAS, 2007).

Os modelos de avaliação de qualidade apresentam diferentes atributos de qualidade em serviços, os quais podem ajudar gestores de empresas na determinação da melhor estratégia na busca da satisfação dos clientes. Porém, cada serviço pode apresentar atributos de qualidade diferentes, de acordo com a especificidade de cada setor e com os tipos de clientes estudados (TINOCO; RIBEIRO, 2008).

\section{Serviços odontológicos}

Segundo Grönroos (2003), um serviço é um fenômeno complicado, pois o termo pode abarcar desde um serviço pessoal até um serviço associado a um produto. Uma definição de serviços de grande aceitação é de que serviço é um processo, consistindo em uma série de atividades mais ou menos intangíveis que são fornecidas como soluções para problemas do cliente (GRÖNROOS, 2003).

A natureza da prestação do serviço odontológico exige a presença do cliente no local da prestação durante todo o tempo da produção, sendo ele corresponsável pelo sucesso do tratamento odontológico, visto que deve seguir procedimentos-padrão de cuidados para o melhor desempenho do serviço. 0 processo de tratamento segue uma sequência que tem início no primeiro contato do cliente com a estrutura da clínica e acaba com a entrega do benefício do serviço. A duração desse processo pode variar entre horas a até meses, dependendo das características de cada caso (SILVEIRA; GROSSI, 2004).

A qualidade e a produtividade dos serviços odontológicos têm relação direta com a demanda, capacidade da clínica e disponibilidade de mão de obra especializada. Uma equipe de apoio atuante e com treinamento adequado juntamente com uma política de marcação de horários e confirmação de consultas são fatores importantes para garantir a produtividade dos serviços odontológicos (SILVEIRA; GROSSI, 2004).

Fruto da relativa saturação do mercado de trabalho para serviços odontológicos e do aumento da preocupação com a qualidade e satisfação dos pacientes por parte das clínicas, muitos profissionais da área vêm utilizando também ferramentas de marketing para conquistar maior sucesso, participação no mercado e novos clientes (BORGES FILHO, 2002). 


\section{Qualidade em serviços odontológicos}

Segundo Borges Filho (2002), o cliente que busca serviços odontológicos, consciente ou inconscientemente, tende a fazer uma avaliação qualitativa da clínica, das pessoas envolvidas e do tratamento recebido. Essa avaliação é estruturada em termos absolutos e relativos, considerando, neste caso, a relação custo-benefício, e dela dependerão o conceito do serviço e a imagem pública do estabelecimento.

Tecnicamente, a avaliação da clínica ou do profissional deveria fundamentar-se na qualidade do serviço recebido. Entretanto, o paciente (cliente) não possui os meios nem o conhecimento para uma avaliação competente. Trata-se de uma avaliação distante de uma apreciação técnica, ou seja, tem muito mais a ver com a maneira como ele é tratado e com as pistas de qualidade que ele vai encontrar no consultório e no profissional do que com a parte técnica. Alguns exemplos de fatores, não técnicos, que influenciam a avaliação da qualidade para serviços odontológicos são: contato físico/telefone com a clínica, aspecto da recepção, do recepcionista e do profissional, temperatura da clínica, limpeza e higiene, comunicação do diagnóstico inicial, orçamento e pontualidade (BORGES FILHO, 2002). Esses atributos também são considerados de relevância para os clientes de serviços médicos ou cuidados à saúde, conforme os estudos de Hanoum, Moses e Noufal (2009).

Ansuj, Zenckner e Godoy (2005) realizaram um levantamento dos atributos de qualidade de serviços odontológicos em clínicas odontológicas particulares de Santa Maria (RS). Os autores fizeram uma pesquisa com 64 clientes das clínicas com o objetivo de determinar a ordem de prioridade dada pelos entrevistados no julgamento da qualidade em serviços odontológicos. Como resultado, os dois principais atributos citados pelos entrevistados são a segurança do profissional quanto ao tratamento e a confiança e precisão na presteza do serviço, atributos estes que podem ser traduzidos como confiabilidade. Outros pontos importantes levantados dizem respeito à limpeza e higiene do local e à pontualidade do atendimento. Esses resultados são confirmados na pesquisa realizada por Garbin et al. (2008), em que a eficiente administração do tempo no consultório além da uniformização do profissional e a humanização do atendimento constituem os itens de maior importância para clientes de serviços odontológicos privados. A pontualidade no atendimento pode ser considerada, pelos clientes de serviços odontológicos, um atributo mais importante do que a competência profissional para a percepção de qualidade (HSU; PAN, 2009).

Dagger, Sweeney e Johnson (2007) descrevem em seu modelo de avaliação da qualidade em serviços de saúde que a qualidade em serviços é avaliada através de múltiplos níveis de abstração e envolve quatro domínios primários, tais como: qualidade interpessoal (envolve atributos que refletem o relacionamento entre o fornecedor de serviço e o cliente); qualidade técnica (compreende as competências técnicas do prestador de serviço); qualidade do ambiente (inclui instalações físicas e características ambientais como atmosfera e tangiveis) e qualidade administrativa (envolve elementos que facilitam a produção do serviço e agregam valor ao cliente). Além dos domínios primários, atributos secundários como interação, relacionamento, resultados, experiência, atmosfera, tangíveis, atualização, operação e suporte interferem na avaliação da qualidade, como mostra a Figura 3.

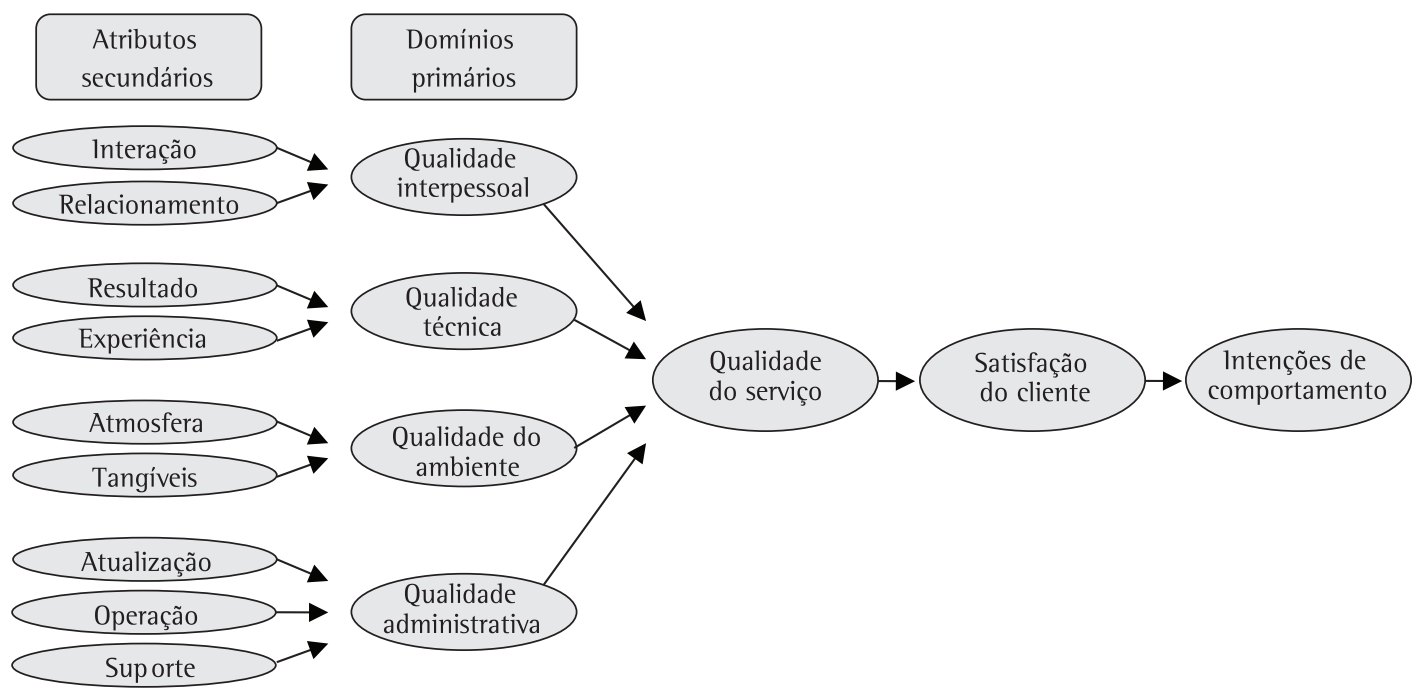

Figura 3. Modelo de avaliação da qualidade em serviços de saúde. Fonte: adaptado de Dagger, Sweeney e Johnson (2007). 
Neste modelo, a qualidade do serviço influencia diretamente a satisfação dos clientes em serviços de saúde. Esse resultado também foi constatado por Ueltschy et al. (2007) em serviços odontológicos no Japão, Estados Unidos e Alemanha.

Devido ao fato de que o modelo de qualidade em serviços de saúde pode ser aplicado a serviços que apresentam características de seguimento ou tratamento e de alto contato e envolvimento dos clientes (DAGGER; SWEENEY; JOHNSON, 2007), ele também pode ser estendido a serviços odontológicos.

\section{Procedimentos metodológicos}

A presente pesquisa pode ser classificada como de natureza aplicada, pois visa contribuir para fins práticos; exploratória, pois envolve levantamento de referencial teórico juntamente com entrevistas a pessoas que possuem experiência relacionada ao assunto abordado; e utiliza-se do levantamento como procedimento técnico, visto que envolve a interrogação direta das pessoas cujo comportamento deseja-se conhecer (SILVA; MENEZES, 2001; CERVO; BERVIAN, 2002).

0 trabalho desenvolvido contemplou o uso da abordagem proposta por Tinoco e Ribeiro (2007) para identificar as relações entre determinantes da satisfação em serviços odontológicos privados. Ele foi realizado em quatro etapas distintas: (i) identificação das relações entre os determinantes da satisfação; (ii) construção do modelo de satisfação; (iii) identificação dos atributos de qualidade; e (iv) hierarquização dos atributos que afetam a qualidade percebida para serviços odontológicos.

\subsection{Etapa 1. Identificação das relações entre os determinantes}

A definição dos determinantes envolve o estudo dos principais modelos e determinantes de satisfação encontrados na literatura e considerados na maioria das pesquisas. Neste artigo serão considerados os seguintes determinantes para a modelagem da satisfação dos clientes, conforme estudo realizado por Tinoco e Ribeiro (2007): desejos pessoais, expectativas, emoções vivenciadas no momento, qualidade percebida, preço do serviço, valor do serviço, desconfirmação das expectativas, imagem corporativa e satisfação dos clientes.

Para a identificação das relações entre os determinantes selecionados para o estudo, foi realizada uma pesquisa descritiva. Ela consistiu de um questionário fechado com nove questões, baseado no instrumento de pesquisa utilizado por Tinoco e
Ribeiro (2007) e apresentado no Anexo 1, no qual o entrevistado deve estabelecer a dependência de cada um dos determinantes em relação aos demais, podendo selecionar uma ou múltiplas alternativas. 0 questionário é acompanhado de uma folha explicativa com as definições de cada determinante (ver quadro 1), para dirimir possíveis dúvidas por parte dos entrevistados e auxiliar na compreensão dos termos abordados.

Conforme a abordagem proposta por Tinoco e Ribeiro (2007), um tamanho de amostra de 40 questionários é suficiente para obter relações significativas com a precisão desejada. Neste trabalho, foi utilizada uma amostra de 50 pessoas, clientes de serviços odontológicos. A pesquisa foi realizada no estado do Rio Grande do Sul, no ano de 2009, abordando indivíduos que utilizam serviços odontológicos, com escolaridade mínima de ensino médio completo. A coleta de dados ocorreu através de entrevistas pessoais. Dentre os entrevistados estão pessoas de ambos os sexos, estudantes universitários, profissionais dos serviços públicos e de empresas privadas, além de alguns aposentados. Atendidos os requisitos de possuir ensino médio concluído e de ser usuário de serviços odontológicos, os respondentes foram selecionados aleatoriamente, de forma a estabelecer uma amostra diversificada no que tange a sexo, idade e classe social.

\subsection{Etapa 2. Construção do modelo de satisfação}

Uma vez aplicado o questionário aos entrevistados, identificou-se a contagem obtida em cada uma das relações entre pares de determinantes. Uma matriz foi construida, a partir das contagens obtidas, na qual o cabeçalho das linhas apresenta os determinantes como variáveis dependentes e o cabeçalho das colunas mostra os mesmos determinantes, mas como variáveis independentes. As relações significativas entre os determinantes foram identificadas através da análise dos resíduos padronizados. No caso de contagens, os resíduos padronizados podem ser calculados através da seguinte equação (AGRESTI; FINLEY, 2009):

$r_{i j}=\frac{f o_{i j}-f e}{\sqrt{f e(1-\% \text { da linha })\left(1-\% \text { coluna }_{j}\right)}}$

Onde, $r_{i j}$ : resíduo padronizado para a contagem observada na relação ij; fo $\mathrm{i}_{\mathrm{ij}}$ : valor observado (no caso, a contagem obtida para cada determinante); fe: valor esperado (no caso, a média dos valores observados).

0 percentual da linha é calculado somando os valores das contagens para cada variável dependente (cabeçalho das linhas) e dividindo esse valor pela 
soma total das contagens. 0 percentual da coluna é calculado da mesma forma, porém, utilizando as contagens das variáveis independentes (cabeçalho das colunas).

Os valores de desvio maiores que 2,0 são os que representam relação significativa entre os respectivos determinantes. Estatisticamente, esse valor é associado a um nível de confiança alfa $=0,05$, o que significa que a probabilidade de um valor de resíduo padronizado maior que $+2,00$ ser obtido ao acaso, sem que exista relação entre os determinantes, é menor que 5\%.

Depois de estabelecidas as relações entre os determinantes da satisfação, foi construído o diagrama de enlace causal, semelhante à Figura 2, o qual representa o modelo de satisfação para o serviço estudado. No diagrama, as setas representam o sentido da influência de um determinante sobre o outro (TINOCO; RIBEIRO, 2007).

\subsection{Etapa 3. Identificação dos atributos de qualidade}

Essa terceira etapa envolveu a identificação dos principais atributos de qualidade que influenciam a qualidade percebida pelos clientes de serviços ondontológicos. Essa identificação foi realizada considerando o referencial teórico sobre a qualidade em serviços odontológicos e brainstorming com usuários desse tipo de serviço, para possibilitar a seleção dos atributos a serem utilizados na pesquisa de hierarquização dos mesmos.

Assim, a partir das pesquisas de Borges Filho (2002), Ansuj, Zenckner e Godoy (2005) e Dagger, Sweeney e Johnson (2007) e de pesquisa aberta com usuários de serviços odontológicos, foram selecionados os atributos apresentados no Quadro 2 para o estudo neste artigo.

\subsection{Etapa 4. Hierarquização dos atributos que afetam a qualidade percebida}

Depois de levantados os atributos que influenciam a percepção dos clientes sobre a qualidade do serviço foi construído um questionário, utilizado como instrumento de pesquisa na última etapa da metodologia. Para tanto, os atributos de qualidade selecionados foram organizados em quatro grupos, seguindo o modelo de avaliação de qualidade em serviços de saúde proposto por Dagger, Sweeney e Johnson (2007). Neste modelo, a avaliação da qualidade ocorre através de quatro domínios primários: qualidade administrativa, qualidade do ambiente, qualidade interpessoal e qualidade técnica. Neste artigo foram realizadas adaptações na terminologia desses grupos ou domínios para facilitar o entendimento do entrevistado. Assim, os atributos identificados na etapa anterior foram agrupados conforme a seguinte designação: (i) aspectos administrativos; (ii) ambiente, mobiliário e equipamentos; (iii) atendimento; e (iv) serviço odontológico.

A hierarquização dos atributos de qualidade identificados e selecionados na etapa anterior foi realizada através da aplicação de uma pesquisa descritiva, com o auxílio do questionário construído, apresentado no Apêndice A. A amostra investigada novamente foi constituída de 50 pessoas, com as mesmas características descritas anteriormente. A pesquisa foi realizada seguindo a mesma sistemática utilizada na pesquisa de satisfação.

A cada entrevistado foi solicitado que assinalasse, de acordo com a importância para cada um, os três principais atributos que afetam a percepção de qualidade dentro de cada um dos quatro grupos que compõem o questionário. Adicionalmente, foi solicitado ao entrevistado que ordenasse a importância dos grupos. Após a conclusão da pesquisa, os dados foram tabulados em uma planilha para a análise.

Quadro 2. Atributos da qualidade selecionados para a pesquisa.

\begin{tabular}{|c|c|}
\hline \multicolumn{2}{|c|}{ Atributos de qualidade para serviços odontológicos } \\
\hline $\begin{array}{l}\text { Confirmação da consulta previamente por parte do recepcionista/ } \\
\text { profissional }\end{array}$ & Simpatia e cordialidade do recepcionista/profissional \\
\hline Disponibilidade de horários para consulta & Pontualidade \\
\hline Disponibilidade de convênios & Atenção dispensada pelo profissional ao cliente \\
\hline Conveniência do local & Sensibilidade do profissional \\
\hline Comunicação clara do diagnóstico e do plano de tratamento & Segurança e tranquilidade transmitidas pelo profissional \\
\hline Contato pós-tratamento & Disponibilidade de estacionamento \\
\hline Decoração do local, música ambiental, climatização e iluminação & Utilização de uniforme, luvas descartáveis e máscara \\
\hline Cadeiras confortáveis & Higienização e esterilização dos materiais \\
\hline Atualização do profissional em métodos e técnicas de tratamento & Avaliação meticulosa, diagnóstico e tratamento apropriados \\
\hline Limpeza do local & Disponibilidade de revistas, jornais e TV \\
\hline $\begin{array}{l}\text { Conforto e tecnologia da cadeira do paciente e equipamentos } \\
\text { associados }\end{array}$ & Informação de métodos de prevenção e manutenção da saúde oral \\
\hline $\begin{array}{l}\text { Condição dos demais equipamentos e materiais utilizados pelo } \\
\text { dentista }\end{array}$ & Referência e/ou parceria com outros especialistas \\
\hline
\end{tabular}


A fase de análise dos dados consistiu em construir a tabela de hierarquização dos atributos que influenciam a qualidade percebida pelos clientes de serviços odontológicos. Nesse sentido, primeiramente foi feita a ordenação final dos quatro grupos de atributos para descobrir a importância deles sobre os atributos de qualidade. Para tanto, foi feita a soma dos inversos das posições de cada grupo e, após, essa soma foi dividida pela soma total dos valores de cada grupo e multiplicada por 100, resultando em um percentual de importância de cada grupo de atributos. Assim, pela ordenação dos percentuais encontrados, obtém-se a ordenação final dos quatro grupos.

0 segundo passo da análise é realizar a contagem das respostas para cada atributo. Depois de realizada a contagem total, o número de respostas de cada atributo é multiplicado pelo percentual de importância calculado para cada grupo, obtendo-se um valor de ordenação para cada atributo individualmente. Dessa forma, foi possível hierarquizar os atributos segundo a importância atribuída pelos clientes de serviços odontológicos.

\section{Resultados}

\subsection{Construção do modelo de satisfação do cliente}

0 modelo de causa e efeito das relações entre os determinantes da satisfação para serviços odontológicos foi criado a partir do resultado da pesquisa descritiva aplicada a clientes do serviço em estudo. Após a coleta dos dados, foi feita a contagem das relações de dependência entre os determinantes, que foram assinaladas na pesquisa. Essa contagem está mostrada na Tabela 1.

A análise da matriz de contagem mostra que 6 respostas apontam que a imagem corporativa da empresa de serviço odontológico depende dos desejos pessoais, 10 respondem que a imagem depende das expectativas, 8 apontam que a imagem depende das emoções, 38 apontam que a imagem depende da qualidade, 9 respondem que a imagem é dependente do preço, 15 marcam a imagem como dependente do valor, 11 apontam que a imagem depende da desconfirmação das expectativas enquanto 28 respondem que a imagem depende da satisfação. Essa leitura pode ser feita para os demais determinantes.

A partir da contagem de respostas (Tabela 1), utilizando a Equação 1 foram calculados os resíduos padronizados que serviram de base para a construção do diagrama de causa e efeito para serviços odontológicos. Os resíduos padronizados são apresentados na Tabela 2, sendo que os valores de desvio maiores que 2,0, destacados nas células hachuradas, representam relação de dependência significativa entre os determinantes, para um nível de confiança de 0,05 , conforme proposto por Tinoco e Ribeiro (2007).

Através da identificação das relações significativas existentes entre os determinantes, foi construído o diagrama de causa e efeito, que representa o modelo de satisfação para serviços odontológicos, apresentados na Figura 4. Nessa figura está ilustrada a intensidade das relações entre os determinantes. Resíduos com valores maiores que $+4,00$ foram apresentados em linha forte; enquanto resíduos com valores entre $+3,00$ e $+4,00$ foram apresentados em linha média e resíduos menores que $+3,00$ em linha fraca.

0 modelo da satisfação construído indica forte inter-relação entre qualidade percebida, desconfirmação das expectativas e satisfação do cliente. Percebe-se um enlace ou feedback entre esses três determinantes, que apresentam os maiores valores residuais, formando a base do modelo de satisfação. Assim, a qualidade percebida e a confirmação das expectativas afetam a satisfação do cliente, e esta, por sua vez, influencia na qualidade percebida e na confirmação das expectativas. Dessa forma, com o aumento da qualidade percebida, aumentaria também a confirmação das expectativas,

Tabela 1. Matriz de contagem de respostas do questionário de determinantes.

\begin{tabular}{|c|c|c|c|c|c|c|c|c|c|c|}
\hline & & \multicolumn{9}{|c|}{ VARIÁVEIS INDEPENDENTES } \\
\hline \multicolumn{2}{|c|}{$\begin{array}{c}\text { SERVIÇOS } \\
\text { ODONTOLÓGICOS }\end{array}$} & Imagem & Desejos & Expectativas & Emoções & Qualidade & Preço & Valor & Desconfirmação & Satisfação \\
\hline \multirow{9}{*}{ 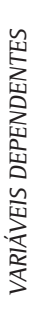 } & lmagem & - & 6 & 10 & 8 & 38 & 9 & 15 & 11 & 28 \\
\hline & Desejos & 13 & - & 29 & 18 & 8 & 7 & 9 & 12 & 11 \\
\hline & Expectativas & 33 & 29 & - & 8 & 15 & 11 & 10 & 3 & 10 \\
\hline & Emoções & 2 & 21 & 17 & - & 30 & 2 & 9 & 29 & 30 \\
\hline & Qualidade & 14 & 10 & 9 & 17 & - & 4 & 19 & 31 & 37 \\
\hline & Preço & 12 & 2 & 8 & 1 & 22 & - & 36 & 5 & 15 \\
\hline & Valor & 4 & 13 & 14 & 6 & 35 & 13 & - & 21 & 31 \\
\hline & Desconfirmação & 5 & 16 & 25 & 18 & 30 & 0 & 9 & - & 38 \\
\hline & Satisfação & 4 & 16 & 21 & 13 & 40 & 9 & 22 & 45 & - \\
\hline
\end{tabular}


Tabela 2. Matriz de resíduos padronizados.

\begin{tabular}{|c|c|c|c|c|c|c|c|c|c|c|}
\hline & & \multicolumn{9}{|c|}{ VARIÁVEIS INDEPENDENTES } \\
\hline \multicolumn{2}{|c|}{$\begin{array}{c}\text { SERVIÇOS } \\
\text { ODONTOLÓGICOS }\end{array}$} & Imagem & Desejos & Expectativas & Emoções & Qualidade & Preço & Valor & Desconfirmação & Satisfação \\
\hline \multirow{9}{*}{ 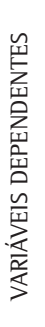 } & lmagem & - & $-2,86$ & $-1,78$ & $-2,28$ & 6,24 & $-1,98$ & $-0,39$ & $-1,52$ & 3,32 \\
\hline & Desejos & $-0,92$ & - & 3,46 & 0,43 & $-2,41$ & $-2,50$ & $-2,03$ & $-1,23$ & $-1,54$ \\
\hline & Expectativas & 4,49 & 3,44 & - & $-2,28$ & $-0,41$ & $-1,44$ & $-1,77$ & $-3,75$ & $-1,83$ \\
\hline & Emoções & $-3,94$ & 1,27 & 0,16 & - & 3,95 & $-3,88$ & $-2,07$ & 3,55 & 3,92 \\
\hline & Qualidade & $-0,66$ & $-1,77$ & $-2,07$ & 0,16 & - & $-3,34$ & 0,72 & 4,12 & 5,94 \\
\hline & Preço & $-0,92$ & $-3,91$ & $-2,31$ & $-4,14$ & 1,60 & - & 5,36 & $-3,17$ & $-0,40$ \\
\hline & Valor & $-3,39$ & $-0,94$ & $-0,67$ & $-2,84$ & 5,40 & $-0,92$ & - & 1,29 & 4,20 \\
\hline & Desconfirmação & $-3,12$ & $-0,12$ & 2,40 & 0,43 & 3,96 & $-4,42$ & $-2,07$ & - & 6,23 \\
\hline & Satisfação & $-3,44$ & $-0,12$ & 1,30 & $-0,95$ & 6,97 & $-2,03$ & 1,58 & 8,19 & - \\
\hline
\end{tabular}
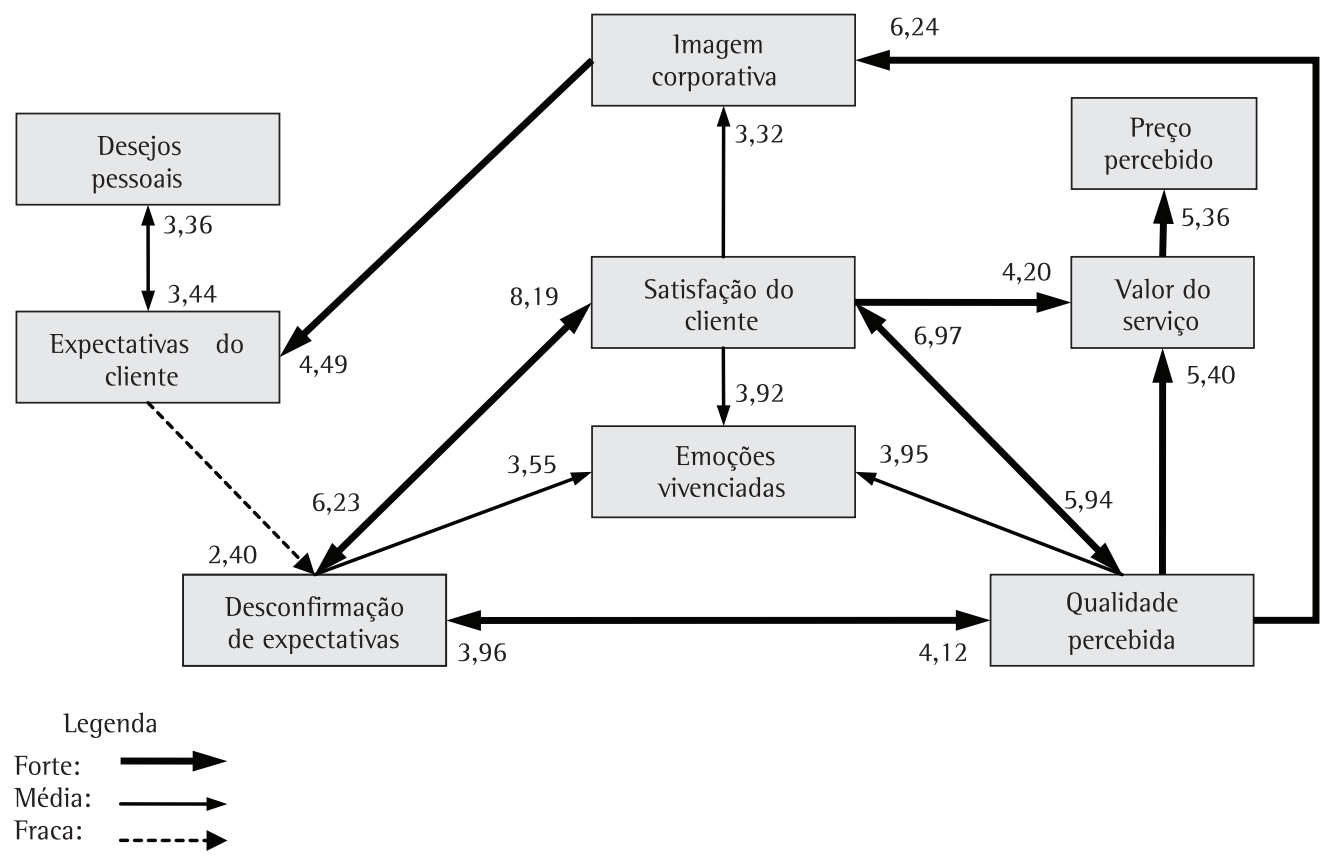

Figura 4. Modelo de causa e efeito da satisfação para serviços odontológicos.

gerando-se a satisfação do cliente. A comparação entre o modelo de satisfação para serviços odontológicos e o modelo de satisfação para serviços médicos (Figura 2) mostra que esse triângulo da qualidade percebida, desconfirmação das expectativas e satisfação do cliente repete-se nos dois modelos.

$A$ análise do modelo construído mostra que a qualidade percebida tem forte relação na criação da imagem corporativa, que, por sua vez, gera as expectativas nos clientes e que, se forem confirmadas, geram satisfação do cliente e qualidade percebida, estabelecendo outro enlace entre os determinantes.

Analisando a Figura 4 também se observa que o valor do serviço é gerado basicamente através da qualidade percebida e da satisfação do cliente, sendo que o valor do serviço é o único determinante que influencia a percepção do preço do serviço, o que mostra a importância da relação custo-benefício para o cliente.

Outras relações mais fracas também aparecem no modelo de satisfação, como a relação mútua de dependência entre desejos pessoais e expectativas e a influência que têm a satisfação, a desconfirmação das expectativas e a qualidade percebida nas emoções vivenciadas pelo cliente do serviço.

\subsection{Hierarquização dos atributos de qualidade}

A hierarquização dos atributos de qualidade para serviços odontológicos foi realizada a partir do resultado da pesquisa descritiva aplicada para identificar essa relação. Após a coleta dos dados, os mesmos foram tabulados em uma planilha eletrônica. 
Primeiramente os quatro grupos de atributos (aspectos administrativos, ambiente, mobiliário e equipamentos, atendimento e serviço odontológico) foram ordenados, através do cálculo do percentual de importância de cada grupo no geral. A Tabela 3 mostra a ordenação final dos grupos.

Após a ordenação dos grupos de atributos foi feito o cálculo do percentual de importância de cada atributo. 0 percentual de importância de cada atributo dentro do grupo foi então multiplicado pelo percentual de importância de seu grupo, obtendo-se o valor final da importância de cada atributo. Baseado nesses valores foi possível ordenar os atributos de acordo com a sua importância para o serviço odontológico. A Tabela 4 mostra a hierarquização final dos atributos de qualidade.

Os resultados são semelhantes aos obtidos por Borges Filho (2002) e Ansuj, Zenckner e Godoy (2005). Alguns atributos citados como importantes pelos autores (limpeza e higiene, comunicação do diagnóstico inicial e orçamento, pontualidade e segurança do profissional) também foram apontados na hierarquização obtida: higienização dos materiais aparece em primeiro lugar, comunicação do diagnóstico fica em quarto, pontualidade em quinto, segurança do profissional em sexto e limpeza em oitavo lugar.
Um destaque importante refere-se ao posicionamento dos atributos ligados à parte técnica do serviço odontológico que aparecem nas primeiras posições: avaliação meticulosa, diagnóstico e tratamento apropriados em segundo lugar, utilização de uniforme, luvas e máscara em terceiro e atualização do profissional em sétimo. Esse posicionamento é reflexo da importância dada pelos clientes ao serviço odontológico propriamente dito em detrimento de aspectos de atendimento, ambiente ou administrativos.

A partir dos resultados obtidos foi possivel elaborar um modelo ampliado que relaciona os determinantes da satisfação do cliente com os principais atributos de qualidade percebida hierarquizados, apresentado na Figura 5. Sendo a qualidade percebida um dos determinantes de maior influência na satisfação do cliente, pode-se concluir que os gestores de serviços odontológicos devem estar atentos aos atributos apontados como de maior importância para a percepção de qualidade dos clientes nessa pesquisa.

Tabela 3. Importância dos grupos de atributos de qualidade.

\begin{tabular}{lc}
\hline \multicolumn{1}{c}{ Grupos } & $\%$ \\
\hline Serviço odontológico & 38,56 \\
Atendimento & 28,00 \\
Ambiente, mobiliário e & 17,44 \\
equipamentos & 16,00 \\
Aspectos administrativos & \\
\hline
\end{tabular}

Tabela 4. Hierarquização dos atributos de qualidade para o serviço odontológico.

\begin{tabular}{|c|c|c|}
\hline Ordem & Atributo & Importância \% \\
\hline 1 & Higienização e esterilização dos materiais & 17,74 \\
\hline 2 & Avaliação meticulosa, diagnóstico e tratamento apropriados & 13,88 \\
\hline 3 & Utilização de uniforme, luvas descartáveis e máscara & 12,72 \\
\hline 4 & Comunicação clara do diagnóstico e do plano de tratamento & 11,76 \\
\hline 5 & Pontualidade & 11,48 \\
\hline 6 & Segurança e tranquilidade transmitidas pelo profissional & 10,64 \\
\hline 7 & Atualização do profissional em métodos e técnicas de tratamento & 10,41 \\
\hline 8 & Limpeza do local & 8,02 \\
\hline 9 & Condição dos demais equipamentos e materiais utilizados pelo dentista & 7,67 \\
\hline 10 & Disponibilidade de horários para consulta & 7,36 \\
\hline 11 & Conforto e tecnologia da cadeira do paciente e equipamentos associados & 6,63 \\
\hline 12 & Disponibilidade de convênios & 6,08 \\
\hline 13 & Conveniência do local & 6,08 \\
\hline 14 & Atenção dispensada pelo profissional ao cliente & 4,20 \\
\hline 15 & Simpatia e cordialidade do recepcionista/profissional & 2,80 \\
\hline 16 & Decoração do local, música ambiental, climatização e iluminação & 2,09 \\
\hline 17 & Informação de métodos de prevenção e manutenção da saúde oral & 1,93 \\
\hline 18 & Disponibilidade de estacionamento & 1,76 \\
\hline 19 & Contato pós-tratamento & 1,76 \\
\hline 20 & Disponibilidade de revistas, jornais e TV & 1,40 \\
\hline 21 & Referência e/ou parceria com outros especialistas & 1,16 \\
\hline 22 & Sensibilidade do profissional & 1,12 \\
\hline 23 & Confirmação da consulta previamente por parte do recepcionista/profissional & 0,96 \\
\hline 24 & Cadeiras confortáveis & 0,35 \\
\hline
\end{tabular}




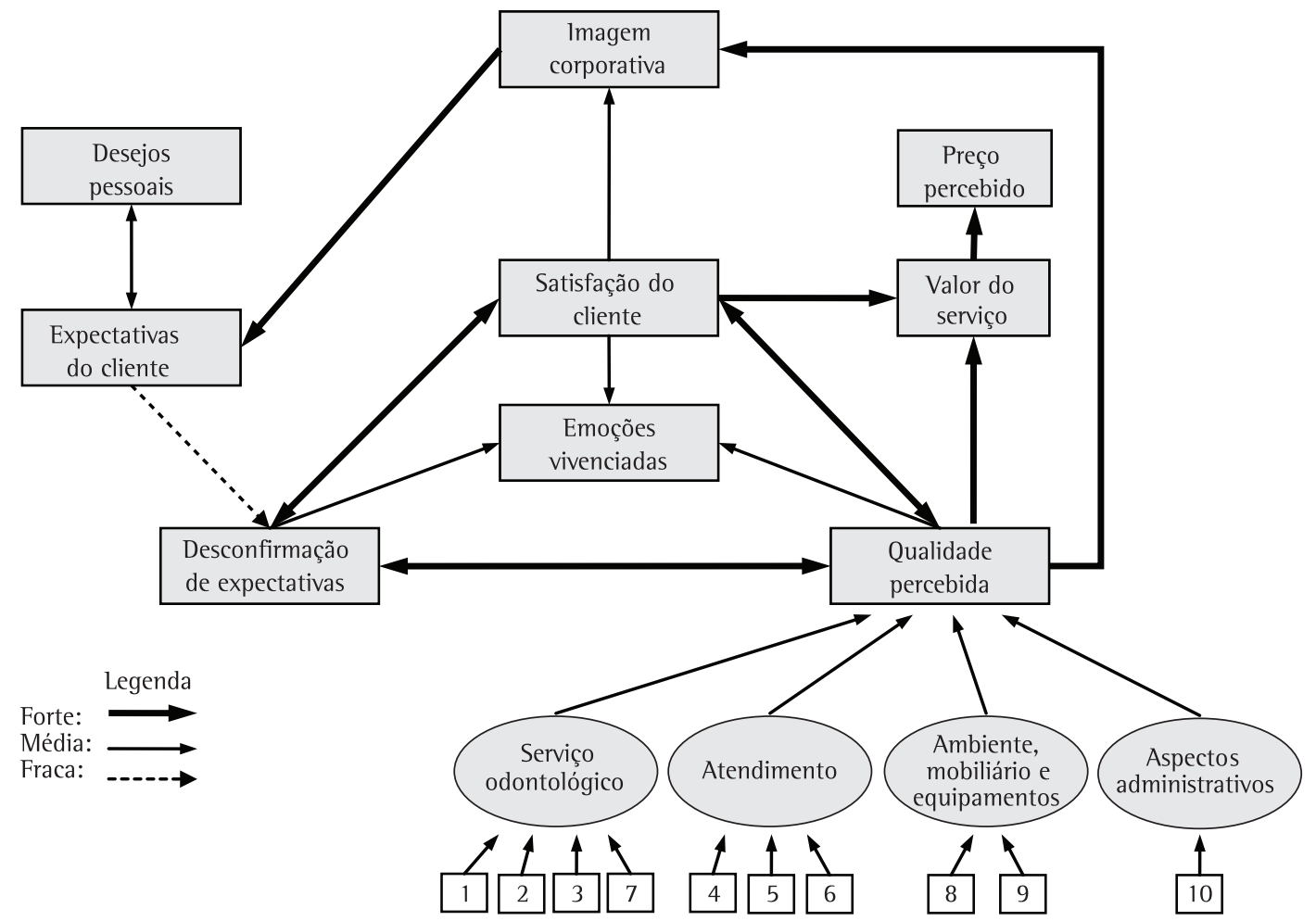

Figura 5. Modelo de causa e efeito da satisfação e qualidade para serviços odontológicos.

\section{Considerações finais}

0 presente artigo teve como objetivos principais a modelagem da satisfação de clientes e a identificação de atributos de qualidade percebida em serviços odontológicos. Nas duas primeiras seções foi feita uma contextualização e uma revisão da literatura abordando conceitos referentes a: satisfação de clientes, modelos e determinantes de satisfação; qualidade em serviços e serviços odontológicos.

0 trabalho aplicado foi organizado em quatro etapas: (1) identificação das relações entre os determinantes e (2) construção do modelo de satisfação de clientes, utilizando a abordagem proposta por Tinoco e Ribeiro (2007) e de uma pesquisa descritiva envolvendo 50 usuários de serviços odontológicos; (3) identificação de atributos de qualidade, através de pesquisa na literatura e brainstorming com usuários do serviço em estudo e (4) hierarquização dos atributos de qualidade percebida identificados na etapa anterior, a partir de outra pesquisa descritiva aplicada a 50 clientes.

A modelagem da satisfação de clientes dos serviços odontológicos permitiu concluir que: (i) há uma forte relação entre a qualidade percebida, a desconfirmação das expectativas e a satisfação do cliente; (ii) o valor do serviço é gerado basicamente da qualidade percebida e da satisfação do cliente; e (iii) há uma forte influência da qualidade percebida sobre a imagem da corporação e desta sobre as expectativas dos clientes.

A hierarquização dos atributos de qualidade mostra que os principais atributos considerados pelos clientes referem-se propriamente ao serviço odontológico, seguidos dos atributos associados a atendimento, ambiente e administrativos. Assim, os principais atributos citados são: (i) higienização e esterilização dos materiais; (ii) avaliação meticulosa, diagnóstico e tratamento apropriados; (iii) utilização de uniforme, luvas descartáveis e máscara; (iv) comunicação clara do diagnóstico e do plano de tratamento; (v) pontualidade; (vi) segurança e tranquilidade transmitidas pelo profissional; (vii) atualização do profissional em métodos e técnicas de tratamento; (viii) limpeza do local; (ix) condição dos demais equipamentos e materiais utilizados pelo dentista; $\mathrm{e}$ (x) disponibilidade de horários para consulta.

A importância do trabalho realizado está na oferta de um modelo para a satisfação de clientes de serviços odontológicos e na identificação dos principais atributos que afetam esse tipo de serviço. Utilizando os resultados apresentados neste artigo, as empresas de serviços odontológicos podem obter melhores resultados, investindo nos elementos mais importantes para o atendimento das necessidades de seus clientes. 
Devido à importância dos atributos técnicos na qualidade percebida pelos clientes de serviços odontológicos, sugere-se para trabalhos futuros a aplicação da pesquisa considerando as diferentes especializações da odontologia, como periodontia, ortodontia, implantodontia, endodontia, entre outras. Assim possíveis ajustes a cada ramo de atuação poderiam ser realizados. Também se sugere a aplicação da pesquisa estratificada em diferentes classes sociais e faixas etárias, tendo em vista um melhor posicionamento no mercado.

\section{Referências}

AGRESTl, A.; FINLEY, B. Statistical Methods for Social Sciences. 4. ed. Prentice Hall, Upper Saddle River, 2009. 609 p.

ANSUJ, A. P.; ZENCKNER, C. L.; GODOY, L. P. Percepção da qualidade dos serviços de odontologia. In: ENCONTRO NACIONAL DE ENGENHARIA DE PRODUÇÃO - ENEGEP, 25., 2005, Porto Alegre. Anais... Porto Alegre: ABEPRO, 2005.

BATESON, J. E. G.; HOFFMAN, K. D. Marketing de serviços. Tradução de Lúcia Simonini. 4. ed. Porto Alegre: Bookman, 2001. 834 p.

BORGES FILHO, A. M. Pontualidade: o diferencial competitivo no atendimento médico-odontológico. Curitiba: Editora Maio, 2002.

CERVO, A. L.; BERVIAN, P. A. Metodologia científica. 5. ed. São Paulo: Prentice Hall, 2002.

CHOWDHARY, N.; PRAKASH, M. Prioritizing service quality dimensions. Managing Service Quality, v. 17, n. 5 , p. 493-509, 2007. http://dx.doi. org/10.1108/09604520710817325

CRONIN, J. J.; TAYLOR, S. A. Measuring Service Quality: a reexamination and extension. Journal of Marketing, v. 56, n. 3, p. 55-68, 1992. http://dx.doi.org/10.2307/1252296

DAGGER, T. S.; SWEENEY, J. C.; JOHNSON, L. W. A hierarchical model of health service quality: scale development and investigation of an integrated model. Journal of Service Research, v. 10, n. 2, p. 123-142, 2007. http://dx.doi. org/10.1177/1094670507309594

EINLOFT, L. Atributos relevantes na satisfação do cliente em unidade de tratamento intensivo pediátrico. 2004. $121 \mathrm{f}$. Dissertação (Mestrado Profissionalizante em Engenharia)Universidade Federal do Rio Grande do Sul, Porto Alegre, 2004.

FITZSIMMONS, J. A.; FITZSIMMONS, M. J. Administração de serviços: operações, estratégia e tecnologia de informação. 2. ed. Porto Alegre: Bookman, 2000.

GARBIN, A. J. l. et al. Marketing em Odontologia: a percepção do cliente em relação ao serviço odontológico de clínica privada. Revista de Odontologia da UNESP, v. 37, n. 2, p. 197-202, 2008.

GIANESI, I. G. N.; CORREA, H. L. Administração estratégica de serviços: operações para a satisfação do cliente. São Paulo: Atlas, 1994.

GRÖNROOS, C. Marketing: gerenciamento e serviços. Tradução de Arlete Simille Marques. 2. ed. Rio de Janeiro: Elsevier, 2003.
HANOUM, S.; MOSES, L. S.; NOUFAL, F. Prioritizing Healthcare Service Attributes: Comparing Importance Performance Analysis and KANO's Model. In: ASIA PACIFIC CONFERENCE ON MANUFACTURING SYSTEM, 2., 2009, Yogyakarta, Indonesia. Proceedings... Yogyakarta, 2009.

HSU, T.; PAN, F. F. C. Application of Monte Carlo AHP in ranking dental quality attributes. Expert Systems with Applications, v. 36, p. 2310-2316, 2009. http://dx.doi. org/10.1016/j.eswa.2007.12.023

HU, H.; KANDAMPULLY, J.; JUWAHEER, T. D. Relationships and impacts of service quality, perceived value, customer satisfaction, and image: an empirical study. The Service Industries Journal, v. 29, n. 2, p. 111-125, 2009. http:// dx.doi.org/10.1080/02642060802292932

INSTITUTO BRASILEIRO DE GEOGRAFIA E ESTATÍSTICA - IBGE. Pesquisa Nacional por Amostra de Domicílios. Rio de Janeiro: IBGE, 2005. Disponível em: $<$ http://www.ibge.gov.br>. Acesso em: 07 out. 2008.

LADHARI, R. A review of twenty years of SERVQUAL research. International Journal of Quality and Service Sciences, v. 1, n. 2, p. 172-198, 2009. http://dx.doi. org/10.1108/17566690910971445

LAl, F., GRIFFIN, M.; BABIN, B. J. How quality, value, image, and satisfaction create loyalty at a Chinese telecom. Journal of Business Research, v. 62, p. 980-986, 2009. http://dx.doi.org/10.1016/j.jbusres.2008.10.015

LIANG, X.; ZHANG, S. Investigation of customer satisfaction in student food service. International Journal of Quality and Service Sciences, v. 1, n. 1, p. 113-124, 2009. http:// dx.doi.org/10.1108/17566690910945903

MARTIN, D. et al. The role of emotion in explaining consumer satisfaction and future behavioural intention. Journal of Services Marketing, v. 22, n. 3, p. 224-236, 2008. http:// dx.doi.org/10.1108/08876040810871183

MIGUEL, P. A. C.; SALOMI, G. E. Uma revisão dos modelos para medição da qualidade de serviços. Revista Produção, v. 14, n. 1, p. 12-30, 2004.

MÜLLER, C. Modelo de gestão integrando planejamento estratégico, sistemas de avaliação de desempenho e gerenciamento de processos (MEIO - Modelo de Estratégia, Indicadores e Operações). 2003. 292 f. Tese (Doutorado em Engenharia de Produção)-Universidade Federal do Rio Grande do Sul, Porto Alegre, 2003.

OLIVER, R. L. A cognitive model of the antecedents and consequences of satisfaction decisions. Journal of Marketing Research, v. 17, p. 460-469, 1980. http:// dx.doi.org/10.2307/3150499

OLIVER, R. L. Cognitive, affective and attribute bases of satisfaction response. Journal of Consumer Research, v. 20, p. 418-430, 1993. http://dx.doi. $\operatorname{org} / 10.1086 / 209358$

OLIVER, R. L. Satisfaction: a behavioral perspective on the consumer. 2. ed. New York: M.E. Sharpe, 2010. 519 p.

PARASURAMAN, A.; ZEITHAML, V. A.; BERRY, L. A conceptual model of service quality and its implications for future research. Journal of Marketing, v. 49, n. 4, p. 41-50, 1985. http://dx.doi.org/10.2307/1251430

RUSSO, F. L. P. Gestão em odontologia. São Paulo: Lovise, 2003.

RUST, R. T.; ZEITHAML, V. A.; LEMON, K. N. O valor do cliente - customer equity: o modelo que está 
reformulando a estratégia corporativa. Porto Alegre: Bookman, 2001.

SCHNEIDER, B.; WHITE, S. S. Service Quality: research perspectives. California: Sage Publications, lnc., 2004. 185 p.

SILVA, E. L.; MENEZES, E. M. Metodologia da pesquisa e elaboração de dissertação. 3. ed. Florianópolis: Laboratório de Ensino à Distância da UFSC, 2001. 121 p.

SILVEIRA, B. L.; GROSSI, R. Aplicação dos 8P's na Gestão Mercadológica de Empresas de Serviços Odontológicos. Think, v. 2, n. 1, p. 76-86, 2004.

SPRENG, R. A.; MACKENZIE, S. B.; OLSHAVSKY, R. W. A reexamination of the determinants of consumer satisfaction. Journal of Marketing, v. 60, n. 3, p. 15-32, 1996. http://dx.doi.org/10.2307/1251839

TINOCO, M. A. C. Proposta de modelos de satisfação para clientes de serviços. 2006. 119 f. Dissertação (Mestrado em Engenharia de Produção)-Universidade Federal do Rio Grande do Sul, Porto Alegre, 2006.

TINOCO, M. A. C.; ARAÚJO, F. C.; RIBEIRO, J. L. D. Proposta de um modelo para a satisfação dos clientes de serviços médicos. In: SEMANA DE ENGENHARIA DE PRODUÇÃO
SUL-AMERICANA - SEPROSUL, 7., 2007, Salto, Uruguai. Anais... Salto: UDELAR, 2007.

TINOCO, M. A. C.; RIBEIRO, J. L. D. Estudo qualitativo dos principais atributos que determinam a percepção de qualidade e de preço dos consumidores de restaurantes à la carte. Revista Gestão e Produção, v. 15, n. 1, p. 73-87, 2008.

TINOCO, M. A. C.; RIBEIRO, J. L. D. Uma nova abordagem para a modelagem das relações entre os determinantes da satisfação dos clientes de serviços. Revista Produção, v. 17, n. 3, p. 454-470, 2007.

UELTSCHY, L. C. et al. Service quality and satisfaction: an international comparison of professional services perceptions. Journal of Services Marketing, v. 21, n. 6, p. 410-423, 2007. http://dx.doi. org/10.1108/08876040710818903

VLACHOS, P. A.; VRECHOPOULOS, A. P. Determinants of behavioral intentions in the mobile internet services market. Journal of Services Marketing, v. 22, n. 4, p. 280-291, 2008. http://dx.doi. org/10.1108/08876040810881687

ZEITHAML, V. A. Marketing de serviços: a empresa com foco no cliente. 2. ed. Porto Alegre: Bookman, 2003. 536 p.

\section{Abstract}

\section{Satisfaction modeling and identification of quality attributes in dental services}

This article presents the construction of a satisfaction model and the identification and ranking of the attributes that affect quality perception of dental service customers. In order to achieve the objectives, two surveys were applied, enabling to identify (i) significant relationships between satisfaction determinants and (ii) the hierarchy of quality attributes. The main contribution of the article is the proposition of a model that helps managers of dental services in their search for quality improvement and customer satisfaction, contributing for companies' development and research in the services sector.

\section{Keywords}

Customer satisfaction. Perceived quality. Dental services. 


\section{ANEXO 1 - QUESTIONÁRIO DE DETERMINANTES DE SATISFAÇÃO}

Tipo de serviço: ODONTOLÓGıICO

Número de questionário:

Com a finalidade de identificar os determinantes principais que afetam a satisfação dos clientes desse tipo de serviço, solicita-se responder o questionário a seguir, selecionando uma, várias ou nenhuma das alternativas:

1. A Imagem corporativa da empresa de serviço depende principalmente de:
a. Desejos pessoais
b. Expectativas
c. Emoções vivenciadas no momento
d. Qualidade percebida
e. Preço do serviço
f. Valor do serviço
g. Confirmação de expectativas
h. Satisfação gerada pelo serviço

2. Os Desejos pessoais dos clientes dependem principalmente de:

a. Imagem corporativa

b. Expectativas

c. Emoções vivenciadas no momento

d. Qualidade percebida

e. Preço do serviço

f. Valor do serviço

g. Confirmação de expectativas

h. Satisfação gerada pelo serviço

3. As Expectativas dos clientes dependem principalmente de:
a. Imagem corporativa
b. Desejos pessoais
c. Emoções vivenciadas no momento
d. Qualidade percebida
e. Preço do serviço
f. Valor do serviço
g. Confirmação de expectativas
h. Satisfação gerada pelo serviço

4. As Emoções vivenciadas no momento dependem principalmente de:

a. Imagem corporativa

b. Desejos pessoais

c. Expectativas

d. Qualidade percebida

e. Preço do serviço

f. Valor do serviço

g. Confirmação de expectativas

h. Satisfação gerada pelo serviço

5. A Qualidade percebida do cliente em relação ao serviço depende principalmente de:

a. Imagem corporativa

b. Desejos pessoais c. Expectativas

d. Emoções vivenciadas no momento

e. Preço do serviço

f. Valor do serviço

g. Confirmação de expectativas

h. Satisfação gerada pelo serviço

6. O Preço do serviço depende principalmente de:
a. Imagem corporativa
b. Desejos pessoais
c. Expectativas
d. Emoções vivenciadas no momento
e. Qualidade percebida
f. Valor do serviço
g. Confirmação de expectativas
h. Satisfação gerada pelo serviço

7. O Valor do serviço depende principalmente de:
a. Imagem corporativa
b. Desejos pessoais
c. Expectativas
d. Emoções vivenciadas no momento
e. Qualidade percebida
f. Preço do serviço
g. Confirmação de expectativas
h. Satisfação gerada pelo serviço

8. A Confirmação de expectativas depende principalmente de:
a. Imagem corporativa
b. Desejos pessoais
c. Expectativas
d. Emoções vivenciadas no momento
e. Qualidade percebida
f. Preço do serviço
g. Valor do serviço
h. Satisfação gerada pelo serviço

9. A Satisfação gerada pelo serviço depende principalmente de:
a. Imagem corporativa
b. Desejos pessoais
c. Expectativas
d. Emoções vivenciadas no momento
e. Qualidade percebida
f. Preço do servico
g. Valor do serviço
h. Confirmação de expectativa 
Apêndice A. Questionário de atributos de qualidade.

Tipo de serviço: ODONTOLÓGıCO

Número de questionário:

Com a finalidade de identificar os atributos mais importantes que afetam a qualidade percebida pelos clientes de serviços odontológicos, solicitamos que você assinale com "X" os três itens mais importantes de cada bloco apresentado a seguir. Agradecemos antecipadamente a sua colaboração. Sua opinião é muito importante para o estudo e melhoria dos serviços odontológicos.

Aspectos administrativos

( ) Disponibilidade de convênios

( ) Disponibilidade de horários para consulta

( ) Confirmação da consulta previamente por parte do recepcionista/profissional

( ) Conveniência do local

( ) Disponibilidade de estacionamento

( ) Contato pós-tratamento

Ambiente, mobiliário e equipamentos

( ) Decoração do local, música ambiental, climatização e iluminação

( ) Cadeiras confortáveis

( ) Disponibilidade de revistas, jornais e TV

( ) Limpeza do local

( ) Conforto e tecnologia da cadeira do paciente e equipamentos associados

( ) Condição dos demais equipamentos e materiais utilizados pelo dentista

Atendimento

( ) Simpatia e cordialidade do recepcionista/profissional

( ) Pontualidade

( ) Atenção dispensada pelo profissional ao cliente

( ) Sensibilidade do profissional

( ) Segurança e tranquilidade transmitidas pelo profissional

( ) Comunicação clara do diagnóstico e do plano de tratamento

Serviço odontológico

( ) Utilização de uniforme, luvas descartáveis e máscara

( ) Higienização e esterilização dos materiais

( ) Avaliação meticulosa, diagnóstico e tratamento apropriados

( ) Atualização do profissional em métodos e técnicas de tratamento

( ) Informação de métodos de prevenção e manutenção da saúde oral

( ) Referência e/ou parceria com outros especialistas

Também se pede que ordene a importância dos grupos abaixo relacionados de 1 a 4, sendo 1 o mais importante, 2 o segundo lugar, e assim por diante:

( ) Aspectos administrativos

( ) Ambiente, mobiliário e equipamentos

( ) Atendimento

( ) Serviço odontológico 